\title{
Assessment of Turbulent Flow Effects on the Vessel Wall Using Four-Dimensional Flow MRI
}

Magnus Ziegler, J onas Lantz, Tino Ebbers and Petter Dyverfeldt

The self-archived version of this journal article is available at Linköping University Electronic Press:

http:/ / urn.kb.se/ resolve?urn=urn:nbn:se:liu:diva-138232

N.B.: When citing this work, cite the original publication.

Ziegler, M., Lantz, J ., Ebbers, T., Dyverfeldt, P., (2017), Assessment of Turbulent Flow Effects on the Vessel Wall Using Four-Dimensional Flow MRI, Magnetic Resonance in Medicine, 77(6), 2310-2319. https:// dx.doi.org/ 10.1002/ mrm.26308

Original publication available at:

https:// dx.doi.org/ 10.1002/ mrm.26308

Copyright: Wiley

http:// eu.wiley.com/WileyCDA/ 


\section{Assessment of Turbulent Flow Effects on the Vessel Wall using 4D Flow MRI}

Magnus Ziegler ${ }^{1,2}$, Jonas Lantz ${ }^{1,2}$, Tino Ebbers ${ }^{1,2}$, Petter Dyverfeldt ${ }^{1,2}$

1 - Division of Cardiovascular Medicine, Department of Medical and Health Sciences, Linköping University, Linköping, Sweden.

2 - Center for Medical Image Science and Visualization (CMIV), Linköping University, Linköping, Sweden.

Division of Cardiovascular Medicine

Department of Medical and Health Sciences

Linköping University

Linköping, 58185

Sweden

Magnus.Ziegler@liu.se

+46101033912

Short Title: Turbulent Flow Effects on Vessel Wall

Word Count: 4150

Disclosures: None 


\section{$\underline{\text { Abstract }}$}

\section{Purpose}

To explore the use of MR-estimated turbulence quantities for the assessment of turbulent flow effects on the vessel wall.

\section{Methods}

Numerical velocity data for two patient-derived models was obtained using computational fluid dynamics (CFD) for two physiological flow rates. 4D Flow MRI measurements were simulated at three different spatial resolutions and used to investigate the estimation of turbulent wall shear stress (tWSS) using the intravoxel standard deviation (IVSD) of velocity and turbulent kinetic energy (TKE) estimated near the vessel wall.

\section{Results}

Accurate estimation of tWSS using the IVSD is limited by the spatial resolution achievable with 4D Flow MRI. TKE, estimated near the wall, has a strong linear relationship to the tWSS (mean $\left.\mathrm{R}^{2}=0.84\right)$. Near-wall TKE estimates from MR simulations have good agreement to CFD-derived ground truth (mean $\mathrm{R}^{2}=0.90$ ). Maps of near-wall TKE have strong visual correspondence to tWSS.

\section{Conclusion}

Near-wall estimation of TKE permits assessment of relative maps of tWSS, but direct estimation of tWSS is challenging due to limitations in spatial resolution. Assessment of tWSS and nearwall TKE may open new avenues for analysis of different pathologies.

Key words: Phase contrast magnetic resonance imaging; wall shear stress; turbulence; turbulent kinetic energy; aorta 


\section{$\underline{\text { Introduction }}$}

Hemodynamic forces acting on the vascular wall appear to affect the risk and progression of several cardiovascular pathologies, such as atherosclerosis and aneurysms $(1,2)$. Of particular interest as disease predictors and descriptors are areas of turbulent flow and their effects on the vessel walls. Turbulent flow near the vessel wall has been associated with adverse vascular remodelling, and damage to the vessel wall (3-10).

An example of pathologically driven remodeling occurs in the endothelial cell layer of the arterial wall. Blood flow causes two forces acting over the surface area of the vessel wall: the normal force causing pressure, and the tangential force causing wall shear stress (WSS). In a region with steady and laminar flow, endothelial cells align along the direction of flow (6). In contrast, turbulent flow fails to align the cells, presumably because the direction, magnitude, and frequency of the shear and pressure stresses are chaotic $(6,7)$. These unaligned cells appear to lose much of their athero-protective capabilities. Additionally, turbulent stresses acting on an atherosclerotic plaque may promote inflammation and erode the plaque surface, making it more vulnerable to rupture (8-10). Unfortunately, non-invasive imaging methods to assess the effects of turbulent flow on the vessel wall are lacking.

In disturbed and turbulent flows, Reynolds decomposition can be used to separate the flow velocity, $U$, into the ensemble-averaged velocity, $\bar{U}$, and the fluctuation of velocity around $\bar{U}, u$ : $U=\bar{U}+u$ (11). In non-pulsatile flows, ensemble-averaging can be obtained by simple timeaveraging, while pulsatile flows require the use of phase-averaging. The latter corresponds well to how the MR signal is acquired in cardiac-gated phase-contrast (PC) MRI(12). The ensembleaveraged standard deviation of $U$ is a commonly used measure of turbulence intensity. Similarly, the WSS can be decomposed into a mean, $\overline{W S S}$, and a fluctuating, wss, component. $\overline{W S S}$ is the aspect of WSS that is estimated by conventional MR velocity mapping. wss, on the other hand, describes rapid and chaotic fluctuations in WSS. The intensity of these chaotic fluctuations can be described by calculating the standard deviation of WSS, and this is referred to as the turbulent wall shear stress (tWSS)(13). 
In vivo measurements concerning the impact of flow on the vessel wall have primarily been performed by estimating $\overline{W S S}$ using 2D cine PC-MRI or three-dimensional, three-directional, cine PC-MRI (4D Flow MRI) velocity data (14-17). As the velocity field measured with 4D Flow MRI represents a mean velocity field, these methods are inherently insensitive to fluctuations in WSS caused by disturbed or turbulent flows. tWSS has only been explored in a small number of computational fluid dynamics (CFD) studies $(13,18)$. Moreover, experimental assessment of the effects of turbulent flow on the vessel wall using non-invasive imaging has not been reported. However, recent developments have extended 4D Flow MRI to permit estimation of velocity fluctuation intensity and turbulent kinetic energy (TKE) $(12,19,20)$. This approach has been validated and demonstrated in a variety of applications (21-25).

Therefore, in an effort to define ways of addressing the impact of turbulent blood flow on the vessel wall, the aim of this work was to investigate the use of MR-estimated turbulence quantities for the assessment of the effects of turbulent flow at the vessel wall.

\section{Methods}

Simulated MR-measurements were performed on numerical velocity data obtained using CFD. In this way, the underlying numerical velocity fields could be used for comparison, thus enabling detailed investigations of the capabilities of MR with respect to the effects of turbulent flow on the vessel wall.

\section{CFD Simulations}

Time-resolved numerical velocity data of non-pulsatile turbulent flow was obtained using CFD simulations for two patient-derived geometries and two flow conditions per geometry. This highresolution numerical velocity data was used as input for simulated 4D Flow MRI measurements and therefore the numerical velocity data also served as ground truth.

The simulations were performed by solving the Navier-Stokes equations using ANSYS CFX 15.0 (ANSYS, Canonsburg, PA, USA) as described previously $(18,26)$. The computational meshes were made using ANSYS ICEM 15.0. Turbulent flow fluctuations were resolved using Large Eddy Simulation (LES), a technique that resolves the larger energy-carrying turbulent scales and 
models smaller isotropic scales where viscous energy dissipation takes place $(13,18,27)$. The unresolved scales are handled with a subgrid-scale model. In this work, the blood flow was resolved by LES using the wall-adapted local eddy viscosity (WALE) subgrid-scale model. The LES technique has been validated through direct numerical simulations (27). Several flowthrough cycles were run to ensure the simulations had reached a steady state before exporting any data, to ensure the transient is excluded from analysis.

Two geometries generated using contrast-enhanced CT or MRI data were used: a patient-specific aortic coarctation (CoA) and a patient-specific aortic stenosis model (AS) with an extended straight outlet region. The AS model features a valve that was constructed to represent a severely constricted case. Both geometries were simulated using two different flow rates, where the slower flow rate is $75 \%$ of the original. These lower flow rate models are therefore denoted AS75 and CoA75, whereas the higher flow rate models are denoted AS100 and CoA100. The AS100 model had a flow rate of $600 \mathrm{ml} / \mathrm{s}$, and the AS75 model had a flow rate of $450 \mathrm{ml} / \mathrm{s}$. The CoA100 model had a flow rate of $300 \mathrm{ml} / \mathrm{s}$, and the CoA75 model had a flow rate of $225 \mathrm{ml} / \mathrm{s}$. In each simulation, a blunt velocity profile was set as the inlet boundary condition, while a constant static pressure was set at the outlet. The walls were rigid, and obeyed the no-slip condition. The fluid modelled was blood with a constant density of $1060 \mathrm{~kg} / \mathrm{m}^{3}$ and constant viscosity $3.5 \cdot 10^{-3} \mathrm{~Pa} \cdot \mathrm{s}$. Both models were simulated using a $50 \mu$ s time step, and exported on at least 20 instances at 20 ms intervals so as to mimic MRI sampling rates. The AS models had a total simulated time of 0.7 seconds, while the CoA models had a total simulated time of 0.5 seconds. An overview of the simulations is presented in Table 1.

\section{D Flow MRI simulations}

The time-resolved numerical velocity data was used to simulate 4D Flow MRI measurements using methods described previously $(28,29)$. In brief, for each model, 3D field-of-views with isotropic voxel sizes of 1,2 , and $2.5 \mathrm{~mm}$ were considered and the velocity distributions $s\left(v_{i}\right)$ in three perpendicular directions $i=\{x, y, z\}$ were obtained by estimating the probability density function derived from the velocities in each voxel using a 3D Gaussian point spread function. The velocity of each computational cell element $j$ within the voxel was weighted with coefficient 
$w_{j}$ based on the cell's distance to the voxel centre: $w_{j}=\frac{\exp \left(-\mathrm{d}_{\mathrm{j}}^{2} / 2 \sigma^{2}\right)}{W}$, where $d_{j}$ is the distance of the $j^{t h}$ cell to the centre of the voxel, $W$ is the sum of the weights within the voxel and $\sigma$ is the variance of the Gaussian function defined from the isotropic spatial resolution $\Delta z$ as $\sigma=\frac{\Delta z}{2.35}$ (30). The signal in each direction $i$ was computed using the Fourier transform of the velocity distribution $s\left(v_{i}\right)$, defined as $S_{i}\left(k_{v}\right)=\int_{-\infty}^{\infty} s\left(v_{i}\right) e^{-i k_{v} v_{i}} d v_{i}$ where $k_{v}=\pi /$ VENC is the applied motion sensitivity. The intra-voxel velocity standard deviation (IVSD) $\sigma_{i}$ can then be derived from the relationship between the magnitudes of the signals measured at two different motion sensitivities, assuming a Gaussian distribution of velocities within the voxel. A non-symmetric flow-encoding scheme was used to obtain measurements at zero motion sensitivity $S_{i}(0)$ and the applied motion sensitivity $k_{v}\left(S_{i}\left(k_{v}\right)\right)$, which allows the estimation of the IVSD as follows:

$$
\sigma_{i}=\left(\frac{1}{k_{v}}\right) \sqrt{2 \ln \left(\mid \frac{S_{i}(0)}{S_{i}\left(k_{v}\right)}\right)}[\mathrm{m} / \mathrm{s}]
$$

The velocity encoding range $(V E N C)$ was set to $150 \mathrm{~cm} / \mathrm{s}$, to optimize sensitivity to the expected range of velocity fluctuations. The fact that the highest velocities in the aortic stenosis and coarctation exceed the VENC value is not a concern as it is the magnitude, not phase, of the MRI signal that is used in IVSD mapping (19). The voxel-wise TKE was computed as: TKE = $\frac{1}{2} \rho \sum_{i=1}^{3} \sigma_{i}^{2}\left[\mathrm{~J} / \mathrm{m}^{3}\right]$ where the density, $\rho$, was set to $1060 \mathrm{~kg} / \mathrm{m}^{3}$.

Mean velocity variations contribute to the intravoxel velocity distribution $s\left(v_{i}\right)$ and therefore the measured IVSD, and typically result in an overestimated IVSD value for the voxel in question. These effects are accounted for by the MR simulation approach used here. Partial volume effects occurring at the interface between blood and static tissue were incorporated into the simulation by modeling the vessel wall as having zero velocity and the same MR signal amplitude as the lumen.

The effect of noise on the results was evaluated for the CoA100 1mm dataset. Noise was incorporated into the simulations by adding a normally distributed random complex noise to the MR signal. Four noise levels were considered, corresponding to signal-to-noise ratios of: 40, 20, 10 , and 5 . 


\section{Assessment of tWSS}

WSS is the stress induced by a fluid moving along a solid boundary, and is given by: $\tau(x)=$ $\mu \frac{\partial u}{\partial x}[P a]$, where $\mu$ is the kinematic viscosity of the fluid, $u$ is the velocity of the fluid, and $x$ is the distance from the wall (31). Similar to the velocity in disturbed and turbulent flows, Reynolds decomposition can be used to decompose WSS into its mean and fluctuating components, yielding $(11,31)$ :

$$
W S S=\overline{W S S}+w S S=\mu \frac{\partial \bar{U}}{\partial x}+\mu \frac{\partial u}{\partial x} .
$$

The standard deviation of WSS is used as a measure of tWSS, i.e.:

$$
t W S S=s t d(W S S)=s t d\left(\mu \frac{\partial \bar{U}}{\partial x}+\mu \frac{\partial u}{\partial x}\right) .
$$

As the standard deviation of the mean WSS is by definition zero, Equation 2 can be simplified to: $t W S S=s t d\left(\mu \frac{\partial u}{\partial x}\right)$. Further, assuming a linear velocity gradient near the wall yields $t W S S=$ std $\left(\mu \frac{u_{2}-u_{1}}{\Delta x}\right)$, where $u_{1}$ is the fluctuating velocity at the wall and $u_{2}$ is the fluctuating velocity at a distance of $\Delta x$ from the wall. Finally, applying the no-slip condition, which implies that $u_{1}=$ 0 , we get that:

$$
t W S S=\mu \frac{s t d\left(u_{2}\right)}{\Delta x},
$$

where $\operatorname{std}\left(u_{2}\right)$ is the IVSD, obtained using Equation 1.

Estimation of $\overline{W S S}$ is known to be limited by the relatively low spatial resolution of 4D Flow MRI $(15,32,33)$. Similarly, given that the size of the viscous sublayer on the vascular wall is likely an order of magnitude smaller than the voxel length, assuming a linear velocity gradient between two adjacent MR voxels at the wall is not be feasible in practice (11). Consequently, we anticipated that tWSS as estimated with Equation 4 would be highly sensitive to the distance from the wall, $\Delta x$. To characterize this constraint, we estimated tWSS using Equation 4 at distances, $\Delta x$, of between $0.05-0.4 \mathrm{~mm}$ from the wall. For this evaluation, $\operatorname{std}\left(u_{2}\right)$ used in Equation 4 was sampled directly from the high-resolution CFD data. This allowed us to isolate the impact of distance without taking spatial averaging into account. This test was performed in the straight extension region of the AS75 model, to minimize geometric complexity. 
MR-based estimation of tWSS was evaluated in the simulated 4D Flow MRI data for each model at voxel sizes of 1,2 , and $2.5 \mathrm{~mm}$. The first voxel along the normal direction of the wall that did not extend into the wall was used to estimate tWSS using Equation 4. The flow direction at the wall was assumed to be the same as the mean flow and the three-directional IVSD was projected onto this direction vector and used to compute tWSS. In this way, the estimated tWSS represents the tWSS component in the main flow direction.

\section{Near-wall TKE estimation}

While the limited spatial resolution of 4D Flow MRI may make the direct estimation of tWSS challenging, the measurement of other turbulence quantities by 4D Flow MRI has previously been shown to be robust. The most commonly used MR-measured turbulence quantity is the TKE. TKE near the vessel wall describes a large proportion of the kinetic energy that is dissipated in the region, along with viscous dissipation. However, MR-estimated TKE near the vessel wall may be affected by the presence of strong velocity gradients, which results in overestimation of the estimated TKE. We therefore investigated the estimation of TKE near the vessel wall, termed near-wall TKE, and its relationship to tWSS.

Near-wall TKE estimation was achieved by sampling the intra-luminal TKE values close to the vessel wall. A near-wall TKE map was created by visiting each wall voxel and calculating the mean TKE value from voxels that: 1) were within a predefined sampling sphere; 2) were inside the vessel; and, 3) were not adjacent to the vessel wall. This sampling approach can also be thought of as convolution with an averaging filter on the segmented volume with wall-adjacent voxels excluded. Sampling in this way ensures that the sampled voxels are in close proximity to the wall voxel of interest, in the lumen, and not strongly affected by partial volume effects. Nearwall TKE estimations were performed for three isotropic voxel sizes $(1,2,2.5 \mathrm{~mm})$. The maximum sampling radius from the centre of the wall voxel was $5.5 \mathrm{~mm}$ for $1 \mathrm{~mm}$ voxels, $7 \mathrm{~mm}$ for $2 \mathrm{~mm}$ voxels, and $8.75 \mathrm{~mm}$ for $2.5 \mathrm{~mm}$ voxels.

\section{Statistical Analysis}

Linear regression analysis was used to describe the relationship between MR-estimated tWSS and CFD-derived true tWSS. Linear regression and Bland-Altman analysis were used to compare 
the MR-simulated near-wall TKE estimate against the corresponding ground-truth near-wall TKE data from CFD using the same sampling method. Linear regression analysis was also used to assess the relationship between near-wall TKE and the ground-truth tWSS, as obtained from the CFD data. For each regression, the coefficient of determination $\left(R^{2}\right)$ was calculated. Intercepts were fixed to zero. The standard error and $P$-value for each regression's slope were also calculated, and $P<0.005$ was considered significant.

\section{$\underline{\text { Results }}$}

The CFD derived $\overline{W S S}$ and tWSS maps for both the CoA and AS geometries are shown in Figure 1 along with scatterplots of ground truth $\overline{W S S}$ vs tWSS for the CoA100 and AS100 models. This figure shows that tWSS is a complementary measure to $\overline{W S S}$, and can in fact be larger than $\overline{W S S}$

The sensitivity of tWSS to distance from the wall, $\Delta x$, is depicted in Figure 2. tWSS was estimated by applying Equation 4 to the high resolution CFD data in the straight portion of the AS75 model. The true value of tWSS is underestimated, and the underestimation becomes progressively worse with increasing distance from the wall (Figure 2A). The estimate-to-truth ratio as a function of distance from the wall, shown in Figure 2B, shows that beyond a distance of $0.15 \mathrm{~mm}$ the estimate is likely less than half of the true value.

Table 2 lists the results of linear regression analysis for MR-estimated tWSS for each model. $\mathrm{R}^{2}$ values range from $0.23-0.60$. Higher spatial resolution tends to result in higher correlations. As expected, the tWSS estimations are underestimates of the true value. The degree of underestimation increases with increased voxel sizes. Figure 3 shows the result for the CoA100 $1 \mathrm{~mm}$ dataset. In this representative result, there is poor visual correspondence to the baseline CFD-derived result, although the post-coarctation dilation has elevated tWSS in both.

Bland-Altman and linear regression analysis showed good agreement between MR-estimated near-wall TKE and CFD-derived ground truth near-wall TKE (Table 3). Across all models and flow rates, the average $\mathrm{R}^{2}$ was $0.90+/-0.01$. On average, 4D Flow MRI slightly overestimated near-wall TKE. The average slope of the regression line was $1.05+/-0.06$. Similarly, the mean 
Bland-Altman bias was $2 \%$ of the maximum MR-estimated near-wall TKE value, with mean limits of agreement of $13 \%$ of the maximum MR-estimated near-wall TKE. Representative Bland-Altman results are shown in Figure 4.

Representative maps of near-wall TKE are shown in Figures 5 and 6 for the AS and CoA models respectively. Visually these results have strong correspondence to the ground truth tWSS results seen in Figure 1. Table 4 lists the results of linear regression analysis for each model. There are strong linear correlations across each flow condition and resolution for both the AS and CoA models. The lowest $R^{2}$ value (0.55) is found within the CoA75 model, and this model also has the largest variation of $R^{2}$ values due to spatial resolution. The AS75 model has the best average $R^{2}$ value (0.95), as well as the smallest variation due to spatial resolution. The slope of the regression line appears to be related to the Reynolds number, as CoA75 has the largest slope and AS100 has the lowest. A similar trend is visible with respect to resolution as the slope decreases with increased voxel size for each model.

Adding noise to the simulated MR data slightly weakened the correlations between near-wall TKE and tWSS in the CoA100 model. $R^{2}$ values for SNR of 40, 20, 10 and 5 were $0.88,0.87$, 0.84 and 0.74 , respectively. This corresponds to decreases in $R^{2}$ of $0.01,0.02,0.06$, and 0.16 when compared to noise-free results.

\section{$\underline{\text { Discussion }}$}

This study investigated MR-based estimation of turbulent flow effects on the vessel wall. While tWSS can theoretically be quantified with MR-measured turbulent velocity fluctuations, this requires spatial resolution beyond what can currently be achieved. However, MR-estimated TKE near to the vessel wall is closely related to tWSS and permits assessment of the effect of turbulent flow on the vessel wall.

The analysis of high-resolution CFD data suggests that a spatial resolution of less than $0.2 \mathrm{~mm}$ is needed in order to have less than 50\% error in the estimated tWSS value. Unfortunately, MRI of the great vessels and heart has voxel sizes that are typically an order of magnitude larger, making accurate estimation of tWSS challenging. 
Estimating tWSS with IVSD values sampled near the vessel wall in simulated 4D Flow MRI data confirmed that currently achievable voxel sizes are insufficient for accurate estimation of tWSS. While the results show visual similarity to the ground truth result, they are severe underestimations of the true tWSS value. The degree of underestimation appears to be related to both the Reynolds number and spatial resolution. Increased underestimations with respect to Reynolds number may be related to the velocity profile of these turbulent flows. In ideal conditions, turbulent flow has a plug-shaped profile with sharp drops near the walls. The viscous sublayer, where these sharp drops in velocity occur, is very small and decreases in size as Reynolds number increases (11,31). Unfortunately, to directly estimate tWSS, the IVSD gradient must be estimated in this narrow region which is not fully resolved by 4D Flow MRI. The severe underestimation in tWSS, as a result of the strong dependency on spatial resolution, limits the applicability of this approach and motivates the use of other turbulence quantities for tWSS estimation.

While direct estimation of tWSS from IVSD estimates requires that the IVSD gradient is linear between the wall and the sampled voxels, the estimation of near-wall TKE is not dependent on such assumptions. The estimation of near-wall TKE appears to be relatively insensitive to spatial resolution. Bland-Altman analysis showed a maximum bias of $9 \%$ of the maximum MRestimated near-wall TKE value, and no clear trend with respect to voxel size. This may be an effect of the size of the sampling region used at each voxel size. The sampling region decreases in maximum voxel count as the voxel size increases, keeping the maximum physical sampling distance similar between different voxel sizes. With respect to spatial resolution, the CoA model appears to be affected more than the AS model. This may be a consequence of the varying lumen diameter in this model, which could cause the fixed-diameter sampling sphere to sample too far into the lumen. This could be alleviated by using an adaptive sampling sphere which changes size with respect to local vessel diameter.

The MR-estimated maps of near-wall TKE visually correspond to the ground truth tWSS result for each model, and the strong linear relationship between near-wall TKE and tWSS appears to hold across geometries, resolutions, and flow rates. Additionally, the relationship between near- 
wall TKE and tWSS was relatively insensitive to noise. The weakest correlations occurred when using the model with the lowest Reynolds number and largest voxel sizes, while the best were found when using higher Reynolds numbers and resolutions. However, the slope of the regression line changes with respect to these factors. There is insufficient data to form conclusions about the sensitivity to these factors, though the slope of the regression line appears most sensitive to geometry.

Previous work has shown that the MR-based $\overline{W S S}$ estimation is sensitive to segmentation inaccuracies and inadequate spatial resolution $(15,32,33)$. As a result, MRI-based $\overline{W S S}$ estimation is inaccurate and should be seen as relative mapping of $\overline{W S S}$. The same sources of error hinder MR-based estimation of absolute tWSS values. However, the near-wall TKE approach presented here allows regions with high and low tWSS to be detected and distinguished. Mapping relative tWSS is useful as regions with high tWSS may not coincide with regions of high $\overline{W S S}$. For example, comparing $\overline{W S S}$ and tWSS ground truth maps from CFD in the CoA model shows that the tWSS is highest in the post-coarctation dilation of the aorta, whereas $\overline{W S S}$ is highest at the inlet of the constriction. Relative tWSS maps can therefore be used in an analogous manner to $\overline{W S S}$ maps. Relative tWSS maps may help describe the effects of local flow conditions like recirculatory zones or eddies which are not captured by $\overline{W S S}$ or OSI.

Recent developments in 4D Flow MRI have presented new flow parameters that enable simple and robust quantification of relevant flow phenomena (34). One such example is flow displacement, which describes the degree of eccentricity of blood flow in a vessel, and therefore reflects a variety of hemodynamic factors including jet impingement and $\overline{W S S}(35,36)$. Similarly, near-wall TKE is a a robust and straightforward parameter that is directly related to tWSS and may also reflect the fluctuating component of pressure that acts at the vessel wall in disturbed and turbulent flows. Future work could include investigating near-wall TKE in patients with aortic aneurysms to assess near-wall TKE as a growth rate predictor, or comparing tWSS maps for different plaque geometries to assess rupture risk.

This study has several limitations. The flow models employed in this study used non-pulsatile Newtonian flow within rigid walls. Assuming non-pulsatile flow results in different flow patterns 
and levels of turbulence. In this scenario, analysis should employ a phase-averaged approach for calculating tWSS and its relationship to near-wall TKE (18). In large vessels such as the aorta, it is both a common and reasonable simplification to model a Newtonian fluid as the effects of a non-Newtonian fluid are likely to be negligible (37). Insufficient sampling of the longer or larger scales of turbulence because of simulation or model constraints may affect estimations of TKE(12). Additionally, this study has been conducted using a limited number of geometries and flow rates and therefore the results must be taken in this context. Further analysis using a wider array of realistic geometries and flow rates would be valuable to characterize the relationship between near-wall TKE and tWSS in different geometries. However, the range of TKE represented by the cases studied here addresses a majority of aortic flow conditions in which the assessment of near-wall TKE may be of interest. Further development of the near-wall TKE estimation method to include weighted sampling or adaptive sizing based on local diameter might improve the results.

In conclusion, this work showed that TKE can be estimated close to the vessel wall using 4D Flow MRI, and the estimated near-wall TKE correlated strongly against tWSS in representative cases of aortic stenosis and aortic coarctation. The proposed near-wall TKE mapping approach is straight-forward to implement in an analysis pipeline, and the possibility to measure near-wall TKE and identify regions of elevated tWSS may open new pathways for understanding pathologically driven vascular remodelling, damage to the endothelial cells, and plaque rupture.

\section{Acknowledgments}

The Swedish National Infrastructure for Computing (SNIC) is acknowledged for computational resources provided by the National Supercomputer Centre (SNIC2014-11-22). Grant support: Swedish Research Council, Medical Research Council of Southeast Sweden (FORSS) 


\section{References}

1. Ernst CCB. Abdominal Aortic Aneurysm. N. Engl. J. Med. 1993;328:1167-1172.

2. Hope MD, Wrenn SJ, Dyverfeldt P. Clinical Applications of Aortic 4D Flow Imaging. Curr. Cardiovasc. Imaging Rep. 2013;6:128-139. doi: 10.1007/s12410-012-9187-8.

3. Khanafer KM, Bull JL, Upchurch GR, Berguer R. Turbulence significantly increases pressure and fluid shear stress in an aortic aneurysm model under resting and exercise flow conditions. Ann. Vasc. Surg. 2007;21:67-74. doi: 10.1016/j.avsg.2006.10.009.

4. Nichols WW, O'Rourke MF. McDonald's Blood Flow in Arteries, Sixth Edition: Theoretical, Experimental and Clinical Principles. 5th ed. London: CRC Press; 2005.

5. Salsac A-V, Sparks SR, Lasheras JC. Hemodynamic changes occurring during the progressive enlargement of abdominal aortic aneurysms. Ann. Vasc. Surg. 2004;18:14-21. doi: 10.1007/s10016-003-0101-3.

6. Davies PF, Remuzzitt A, Gordon EJ, Dewey CF, Gimbrone MA, Remuzzi A. Turbulent fluid shear stres induces vascular endothelial cell turnover in vivo. Proc. Natl. Acad. Sci. USA 1986;83:2114-2117.

7. Davies P. Flow-mediated endothelial mechanotransduction. Physiol. Rev. 1995;75:519-560.

8. Loree H, Kamm R. Turbulent pressure fluctuations on surface of model vascular stenoses. Am. Physiol. Soc. 1991;261:H644-H650.

9. McGraw AP, Bagley J, Chen W-S, Galayda C, Nickerson H, Armani A, Caprio M, Carmeliet $\mathrm{P}$, Jaffe IZ. Aldosterone increases early atherosclerosis and promotes plaque inflammation through a placental growth factor-dependent mechanism. J. Am. Heart Assoc. 2013;2:e000018. doi: 10.1161/JAHA.112.000018.

10. Sameshima N, Yamashita A, Sato S, Matsuda S, Matsuura Y, Asada Y. The Values of Wall Shear Stress, Turbulence Kinetic Energy and Blood Pressure Gradient are Associated with Atherosclerotic Plaque Erosion in Rabbits. J. Atheroscler. Thromb. 2014;21:831-8.

11. Mathieu J, Scott J. An Introduction to Turbulent Flow. Cambridge University Press; 2000.

12. Dyverfeldt P, Gårdhagen R, Sigfridsson A, Karlsson M, Ebbers T. On MRI turbulence 
quantification. Magn. Reson. Imaging 2009;27:913-22. doi: 10.1016/j.mri.2009.05.004.

13. Gårdhagen R, Lantz J, Carlsson F, Karlsson M. Quantifying turbulent wall shear stress in a stenosed pipe using large eddy simulation. J. Biomech. Eng. 2010;132:061002. doi: 10.1115/1.4001075.

14. Stalder AF, Russe MF, Frydrychowicz A, Bock J, Hennig J, Mark1 M. Quantitative 2D and 3D phase contrast MRI: optimized analysis of blood flow and vessel wall parameters. Magn. Reson. Med. 2008;60:1218-31. doi: 10.1002/mrm.21778.

15. Potters W V, van Ooij P, Marquering H, Vanbavel E, Nederveen AJ. Volumetric arterial wall shear stress calculation based on cine phase contrast MRI. J. Magn. Reson. Imaging 2014;00:112. doi: $10.1002 / \mathrm{jmri} .24560$.

16. Osinnski JN, Ku DN, Mukundan S, Loth F, Pettigrew RI. Determination of wall shear stress in the aorta with the use of MR phase velocity mapping. J. Magn. Reson. Imaging 1995;5:640647. doi: 10.1002/jmri.1880050605.

17. Oyre S, Pedersen EM, Ringgaard S, Boesiger P, Paaske WP. In vivo wall shear stress measured by magnetic resonance velocity mapping in the normal human abdominal aorta. Eur. J. Vasc. Endovasc. Surg. 1997;13:263-271. doi: 10.1016/S1078-5884(97)80097-4.

18. Lantz J, Gårdhagen R, Karlsson M. Quantifying turbulent wall shear stress in a subject specific human aorta using large eddy simulation. Med. Eng. Phys. 2012;34:1139-48. doi: 10.1016/j.medengphy.2011.12.002.

19. Dyverfeldt P, Sigfridsson A, Kvitting JPE, Ebbers T. Quantification of intravoxel velocity standard deviation and turbulence intensity by generalizing phase-contrast MRI. Magn. Reson. Med. 2006;56:850-858. doi: 10.1002/mrm.21022.

20. Dyverfeldt P, Kvitting J-PE, Sigfridsson A, Engvall J, Bolger AF, Ebbers T. Assessment of fluctuating velocities in disturbed cardiovascular blood flow: in vivo feasibility of generalized phase-contrast MRI. J. Magn. Reson. Imaging 2008;28:655-63. doi: 10.1002/jmri.21475.

21. Dyverfeldt P, Hope MD, Tseng EE, Saloner D. Magnetic resonance measurement of turbulent kinetic energy for the estimation of irreversible pressure loss in aortic stenosis. JACC.

Cardiovasc. Imaging 2013;6:64-71. doi: 10.1016/j.jcmg.2012.07.017. 
22. Zajac J, Eriksson J, Dyverfeldt P, Bolger AF, Ebbers T, Carlhäll C-J. Turbulent kinetic energy in normal and myopathic left ventricles. J. Magn. Reson. Imaging 2015;41:1021-1029. doi: $10.1002 /$ jmri.24633.

23. Dyverfeldt P, Kvitting J-PE, Carlhäll CJ, Boano G, Sigfridsson A, Hermansson U, Bolger AF, Engvall J, Ebbers T. Hemodynamic aspects of mitral regurgitation assessed by generalized phase-contrast MRI. J. Magn. Reson. Imaging 2011;33:582-8. doi: 10.1002/jmri.22407.

24. Arzani A, Dyverfeldt P, Ebbers T, Shadden SC. In vivo validation of numerical prediction for turbulence intensity in an aortic coarctation. Ann. Biomed. Eng. 2012;40:860-70. doi: 10.1007/s 10439-011-0447-6.

25. Lantz J, Ebbers T, Engvall J, Karlsson M. Numerical and experimental assessment of turbulent kinetic energy in an aortic coarctation. J. Biomech. 2013;46:1851-8. doi: 10.1016/j.jbiomech.2013.04.028.

26. Andersson M, Lantz J, Ebbers T, Karlsson M. Quantitative Assessment of Turbulence and Flow Eccentricity in an Aortic Coarctation: Impact of Virtual Interventions. Cardiovasc. Eng. Technol. 2015. doi: 10.1007/s13239-015-0218-x.

27. Gårdhagen R, Lantz J, Carlsson F, Karlsson M. Large Eddy Simulation of Stenotic Flow for Wall Shear Stress Estimation - Validation and Application. WSEAS Trans. Biol. Biomed. $2011 ; 8$.

28. Petersson S, Dyverfeldt P, Gårdhagen R, Karlsson M, Ebbers T. Simulation of phase contrast MRI of turbulent flow. Magn. Reson. Med. 2010;64:1039-46. doi: 10.1002/mrm.22494.

29. Casas B, Lantz J, Dyverfeldt P, Ebbers T. 4D flow MRI-Based pressure loss estimation in stenotic flows: Evaluation using numerical simulations. Magn. Reson. Med. 2015. doi: $10.1002 / \mathrm{mrm} .25772$.

30. Haacke EM, Brown RW, Thompson MR, Venkatesan R. Magnetic Resonance Imaging: Physical Principles and Sequence Design. Wiley; 1999.

31. Çengel YA, Cimbala JM. Fluid Mechanics: Fundamentals and Applications. McGrawHillHigher Education; 2006.

32. Petersson S, Dyverfeldt P, Ebbers T. Assessment of the accuracy of MRI wall shear stress 
estimation using numerical simulations. J. Magn. Reson. Imaging 2012;36:128-38. doi: 10.1002/jmri.23610.

33. Potters W V., Marquering H a., VanBavel E, Nederveen AJ. Measuring Wall Shear Stress Using Velocity-Encoded MRI. Curr. Cardiovasc. Imaging Rep. 2014;7:9257. doi: $10.1007 / \mathrm{s} 12410-014-9257-1$.

34. Hope MD, Sedlic T, Dyverfeldt P. Cardiothoracic magnetic resonance flow imaging. J. Thorac. Imaging 2013;28:217-30. doi: 10.1097/RTI.0b013e31829192a1.

35. Sigovan M, Hope MD, Dyverfeldt P, Saloner D. Comparison of four-dimensional flow parameters for quantification of flow eccentricity in the ascending aorta. J. Magn. Reson. Imaging 2011;34:1226-1230. doi: 10.1002/jmri.22800.

36. Hope MD, Meadows AK, Hope T a, Ordovas KG, Saloner D, Reddy GP, Alley MT, Higgins CB. Clinical evaluation of aortic coarctation with 4D flow MR imaging. J. Magn. Reson. Imaging 2010;31:711-8. doi: 10.1002/jmri.22083.

37. Ku DN. Blood Flow in Arteries. Annu. Rev. Fluid Mech. 1997;29:399-434. doi: 10.1146/annurev.fluid.29.1.399. 
Table 1 - Summary of simulated models

\begin{tabular}{|c|c|c|c|c|}
\hline Model & \# Cells & Inlet Re & Inlet flow rate (ml/s) & Total Time/Step \\
\hline \multirow{2}{*}{ CoA } & \multirow{2}{*}{$3.1 \mathrm{M}$} & 3000 & 225 & \multirow{2}{*}{$0.5 \mathrm{sec} / 0.02$} \\
\cline { 3 - 4 } $\mathrm{AS}$ & \multirow{2}{*}{$3.2 \mathrm{M}$} & 6000 & 300 & \multirow{2}{*}{$0.7 \mathrm{sec} / 0.02$} \\
\cline { 3 - 4 } & & 9000 & 450 & \\
\hline
\end{tabular}


Table 2 - Results of linear regression between MR-estimated tWSS and ground truth CFDderived tWSS

\begin{tabular}{|c|c|c|c|}
\hline \multirow{3}{*}{ Model } & $\begin{array}{c}\text { Resolution } \\
{[\mathbf{m m}]}\end{array}$ & Slope (+/- SE) & $\mathbf{R}^{2}$ \\
\hline \multirow{3}{*}{ CoA75 } & 1 & $16.39+/-7.10 \mathrm{E}-2$ & 0.54 \\
\cline { 2 - 4 } & 2 & $21.66+/-3.10 \mathrm{E}-1$ & 0.23 \\
\cline { 2 - 4 } CoA100 & 2.5 & $32.38+/-5.05 \mathrm{E}-1$ & 0.30 \\
\cline { 2 - 4 } & 1 & $24.57+/-9.00 \mathrm{E}-1$ & 0.59 \\
\cline { 2 - 4 } & 2.5 & $39.46+/-4.12 \mathrm{E}-1$ & 0.35 \\
\hline \multirow{3}{*}{ AS75 } & 1 & $24.42+/-9.64 \mathrm{E}-2$ & 0.55 \\
\cline { 2 - 4 } & 2 & $72.77+/-4.45 \mathrm{E}-1$ & 0.57 \\
\cline { 2 - 4 } & 2.5 & $101.04+/-7.31 \mathrm{E}-1$ & 0.59 \\
\hline \multirow{3}{*}{ AS100 } & 1 & $36.08+/-1.25 \mathrm{E}-1$ & 0.49 \\
\cline { 2 - 4 } & 2 & $88.90+/-5.82 \mathrm{E}-1$ & 0.51 \\
\cline { 2 - 4 } & 2.5 & $125.71+/-9.41 \mathrm{E}-1$ & 0.54 \\
\hline
\end{tabular}


Table 3 - Results of linear regression and Bland-Altman analysis between MR-estimated near-wall TKE and CFD derived near-wall TKE.

\begin{tabular}{|c|c|c|c|c|c|}
\cline { 2 - 6 } \multicolumn{1}{c|}{} & \multicolumn{3}{c|}{ Linear Regression } & \multicolumn{2}{c|}{ Bland-Altman } \\
\hline \multirow{3}{*}{ Model } & $\begin{array}{c}\text { Resolution } \\
{[\mathbf{m m}]}\end{array}$ & Slope (+/- SE) & $\mathbf{R}^{2}$ & Bias* $^{*}$ & $\begin{array}{c}\text { Limits of } \\
\text { Agreement* }\end{array}$ \\
\hline \multirow{4}{*}{ CoA75 } & 1 & $1.10+/-1.86 \mathrm{E}-3$ & 0.91 & $4 \%$ & $10 \%$ \\
\cline { 2 - 6 } & 2 & $1.27+/-8.38 \mathrm{E}-3$ & 0.73 & $9 \%$ & $20 \%$ \\
\cline { 2 - 6 } & 2.5 & $1.40+/-1.10 \mathrm{E}-2$ & 0.74 & $9 \%$ & $17 \%$ \\
\hline \multirow{3}{*}{ AS75 } & 1 & $1.02+/-9.91 \mathrm{E}-4$ & 0.97 & $2 \%$ & $6 \%$ \\
\cline { 2 - 6 } & 2 & $1.29+/-5.94 \mathrm{E}-3$ & 0.84 & $7 \%$ & $13 \%$ \\
\cline { 2 - 6 } & 2.5 & $1.25+/-5.13 \mathrm{E}-3$ & 0.90 & $6 \%$ & $11 \%$ \\
\cline { 2 - 6 } & 2 & $0.91+/-7.20 \mathrm{E}-4$ & 0.97 & $-2 \%$ & $9 \%$ \\
\hline \multirow{3}{*}{ AS100 } & 2.5 & $0.97+/-1.23 \mathrm{E}-3$ & 0.98 & $0 \%$ & $6 \%$ \\
\cline { 2 - 6 } & 1 & $0.78+/-1.01 \mathrm{E}-3$ & 0.92 & $-6 \%$ & $25 \%$ \\
\cline { 2 - 6 } & 2 & $0.83+/-1.70 \mathrm{E}-3$ & 0.95 & $-4 \%$ & $18 \%$ \\
\hline
\end{tabular}

* - Bland-Altman bias and limits of agreement are normalized to maximum 4D Flow MRI measurement. 
Table 4 - Results of linear regression between near-wall TKE and tWSS.

\begin{tabular}{|c|c|c|c|}
\hline \multirow{3}{*}{ Model } & $\begin{array}{c}\text { Resolution } \\
{[\mathbf{m m}]}\end{array}$ & Slope (+/- SE) & $\mathbf{R}^{2}$ \\
\hline \multirow{3}{*}{ CoA75 } & 1 & $0.1287+/-2.90 \mathrm{E}-4$ & 0.83 \\
\cline { 2 - 4 } & 2 & $0.0873+/-6.77 \mathrm{E}-4$ & 0.55 \\
\cline { 2 - 4 } CoA100 & 2.5 & $0.0835+/-7.25 \mathrm{E}-4$ & 0.60 \\
\cline { 2 - 4 } & 1 & $0.0959+/-1.60 \mathrm{E}-4$ & 0.90 \\
\hline \multirow{3}{*}{ AS75 } & 2 & $0.0787+/-3.74 \mathrm{E}-4$ & 0.77 \\
\cline { 2 - 4 } & 2.5 & $0.0731+/-3.82 \mathrm{E}-4$ & 0.81 \\
\cline { 2 - 4 } & 2.5 & $0.0607+/-7.18 \mathrm{E}-5$ & 0.94 \\
\hline \multirow{3}{*}{ AS100 } & 1 & $0.0552+/-1.19 \mathrm{E}-4$ & 0.95 \\
\cline { 2 - 4 } & 2 & $0.0538+/-1.28 \mathrm{E}-4$ & 0.93 \\
\cline { 2 - 4 } & 2.5 & $0.0520+/-1.56 \mathrm{E}-4$ & 0.93 \\
\hline
\end{tabular}




\section{FIGURE CAPTIONS}

Figure 1 - Ground truth $\overline{W S S}$ (A) and tWSS (B) for the aortic coarctation model, and $\overline{W S S}$ (D) and tWSS (E) for the aortic stenosis model. Ground truth $\overline{W S S}$ vs tWSS for the CoA100 model (C) and the AS100 model (F).

Figure 2 - Agreement between CFD-derived ground truth tWSS and tWSS estimated using the high resolution CFD data for different distances $\Delta \mathrm{x}$. A) Scatter plots. B) Estimation-to-truth ratio (estimate / truth) versus distance from wall. Colors represent nodes at different distances from the wall. The black line in $(\mathrm{A})$ is the identity line.

Figure 3 - Representative MR-estimated tWSS results for CoA model. (A) shows the mapped result for the CoA100 1mm model, and (B) shows the correlation between the MR-estimated result and the ground truth CFD-derived result for the same model.

Figure 4 - Bland-Altman analysis of the MR-estimated near-wall TKE versus the CFD near-wall TKE in the CoA100 2.5mm model. There is good agreement with CFD, with a bias +/- 2SD of $15.4+/-31.7\left[\mathrm{~J} / \mathrm{m}^{3}\right]$. The linear regression had a slope of $1.25(\mathrm{p}<0.0001)$ and $\mathrm{R}^{2}$ of 0.90 .

Figure 5 - Representative near-wall TKE estimation results for the AS100 model at spatial resolutions of $1 \mathrm{~mm}(\mathrm{~A} \& \mathrm{~B})$ and $2.5 \mathrm{~mm}(\mathrm{C} \& \mathrm{D})$. A \& $\mathrm{C}$ show maps of near-wall TKE while $\mathrm{B}$ $\&$ D show linear regression results for MR-estimated near-wall TKE versus CFD derived tWSS. The linear regression at $1 \mathrm{~mm}$ had $\mathrm{R}^{2}=0.90$, and $\mathrm{R}^{2}=0.93$ at $2.5 \mathrm{~mm}$.

Figure 6 - Representative near-wall TKE estimation results for the CoA100 model at spatial resolutions of $1 \mathrm{~mm}(\mathrm{~A} \& \mathrm{~B})$ and $2.5 \mathrm{~mm}(\mathrm{C} \& \mathrm{D})$. A \& C show maps of near-wall TKE while B \& D show linear regression results for MR-estimated near-wall TKE versus CFD derived tWSS. The linear regression at $1 \mathrm{~mm}$ had $\mathrm{R}^{2}=0.90$, and $\mathrm{R}^{2}=0.81$ at $2.5 \mathrm{~mm}$. 

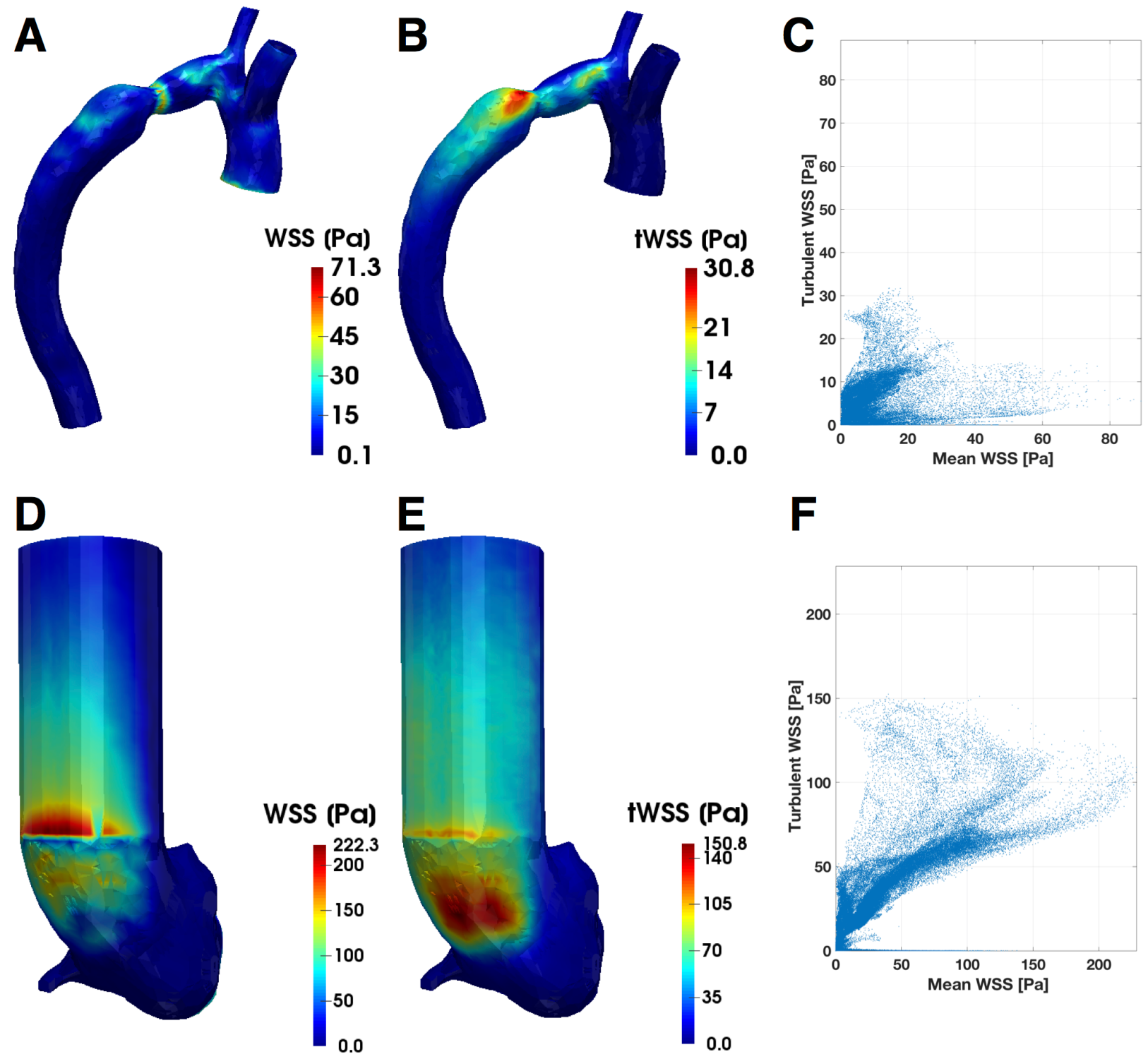

Figure 1 - Ground truth $\overline{W S S}$ (A) and tWSS (B) for the aortic coarctation model, and $\overline{W S S}$ (D) and tWSS (E) for the aortic stenosis model. Ground truth $\overline{W S S}$ vs tWSS for the CoA100 model (C) and the AS100 model (F). 

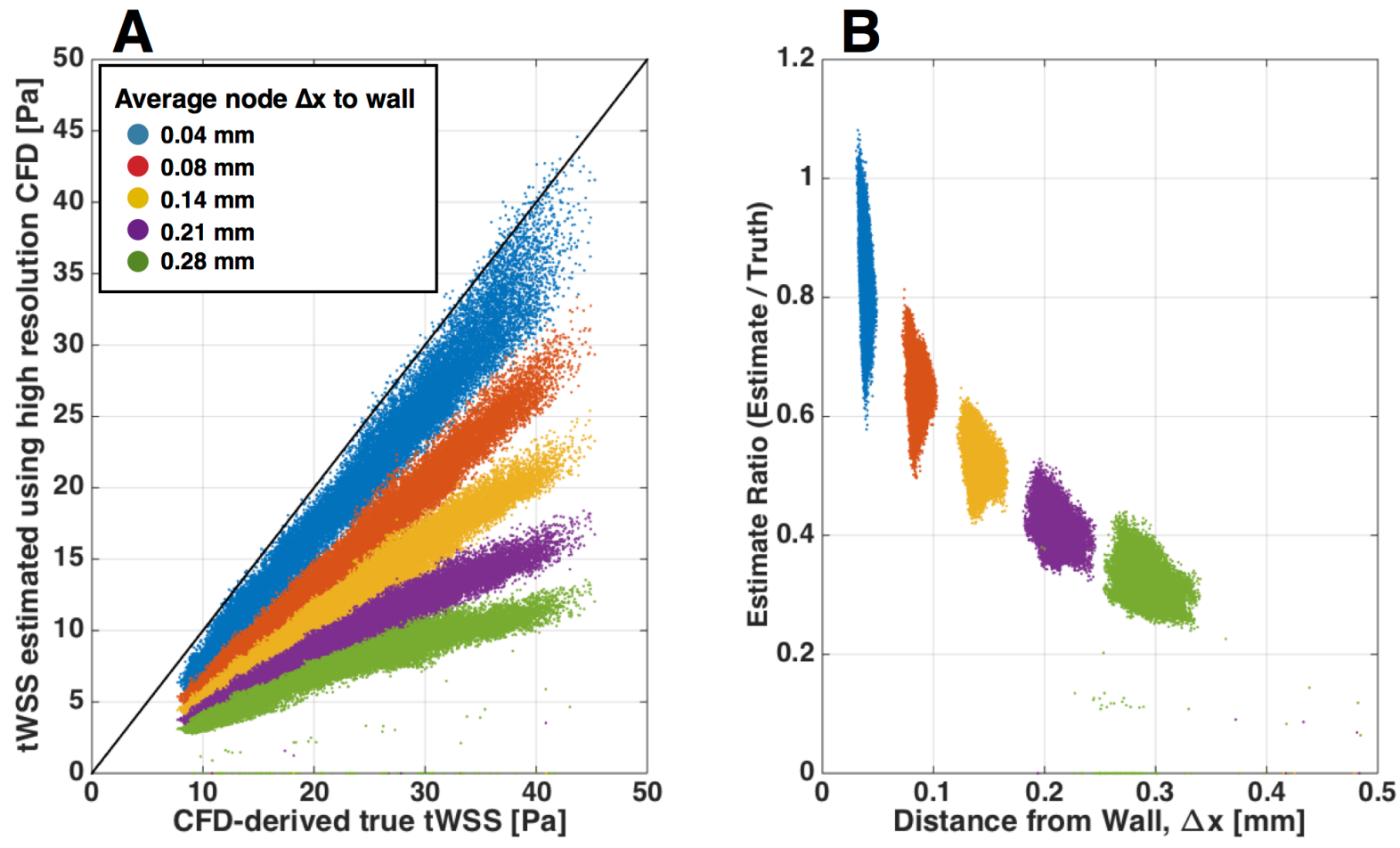

Figure 2 - Agreement between CFD-derived ground truth tWSS and tWSS estimated using the high resolution CFD data for different distances $\Delta \mathrm{x}$. A) Scatter plots. B) Estimation-to-truth ratio (estimate / truth) versus distance from wall. Colors represent nodes at different distances from the wall. The black line in $(\mathrm{A})$ is the identity line. 

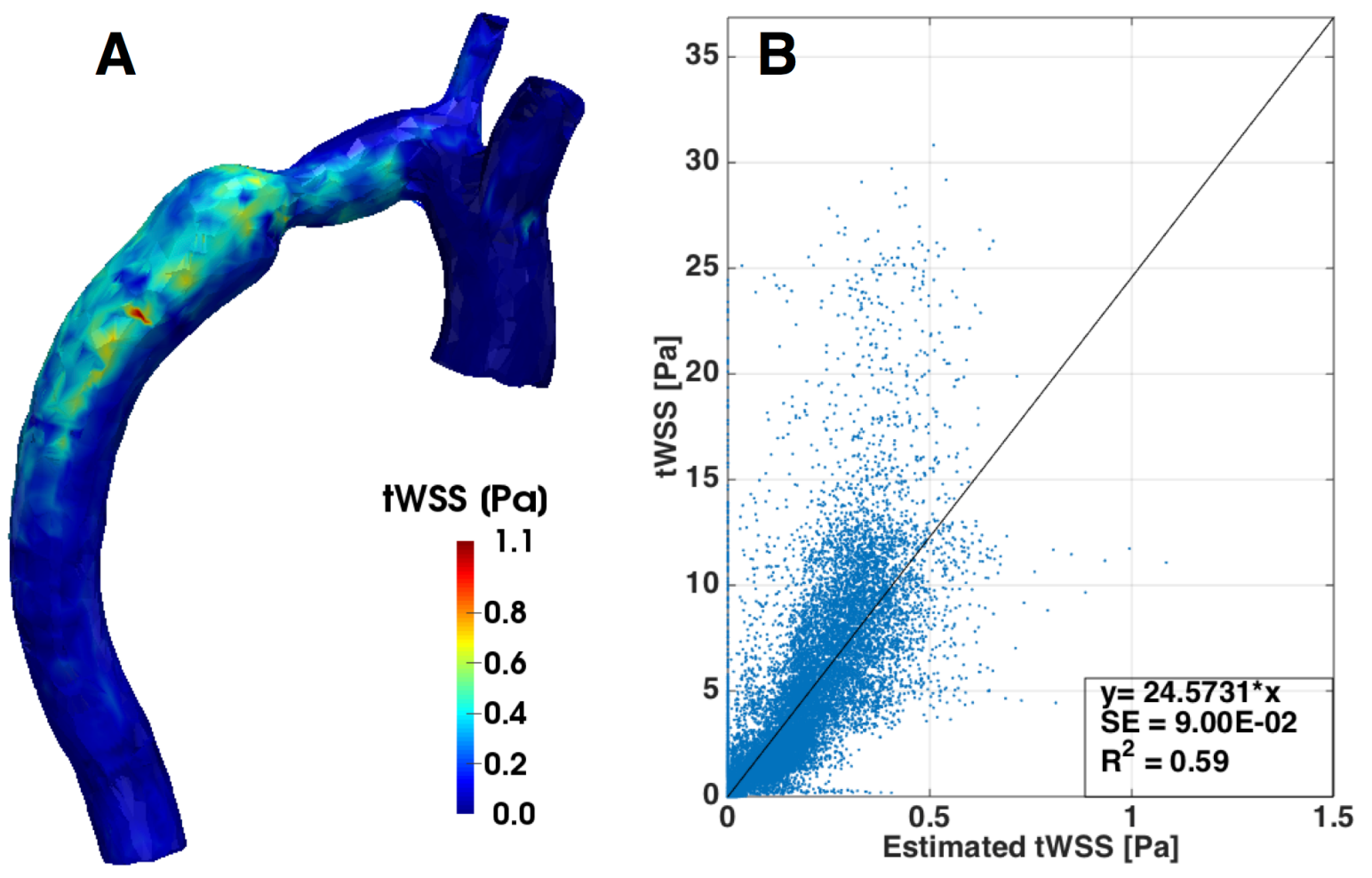

Figure 3 - Representative MR-estimated tWSS results for CoA model. (A) shows the mapped result for the CoA100 1mm model, and (B) shows the correlation between the MR-estimated result and the ground truth CFD-derived result for the same model. 

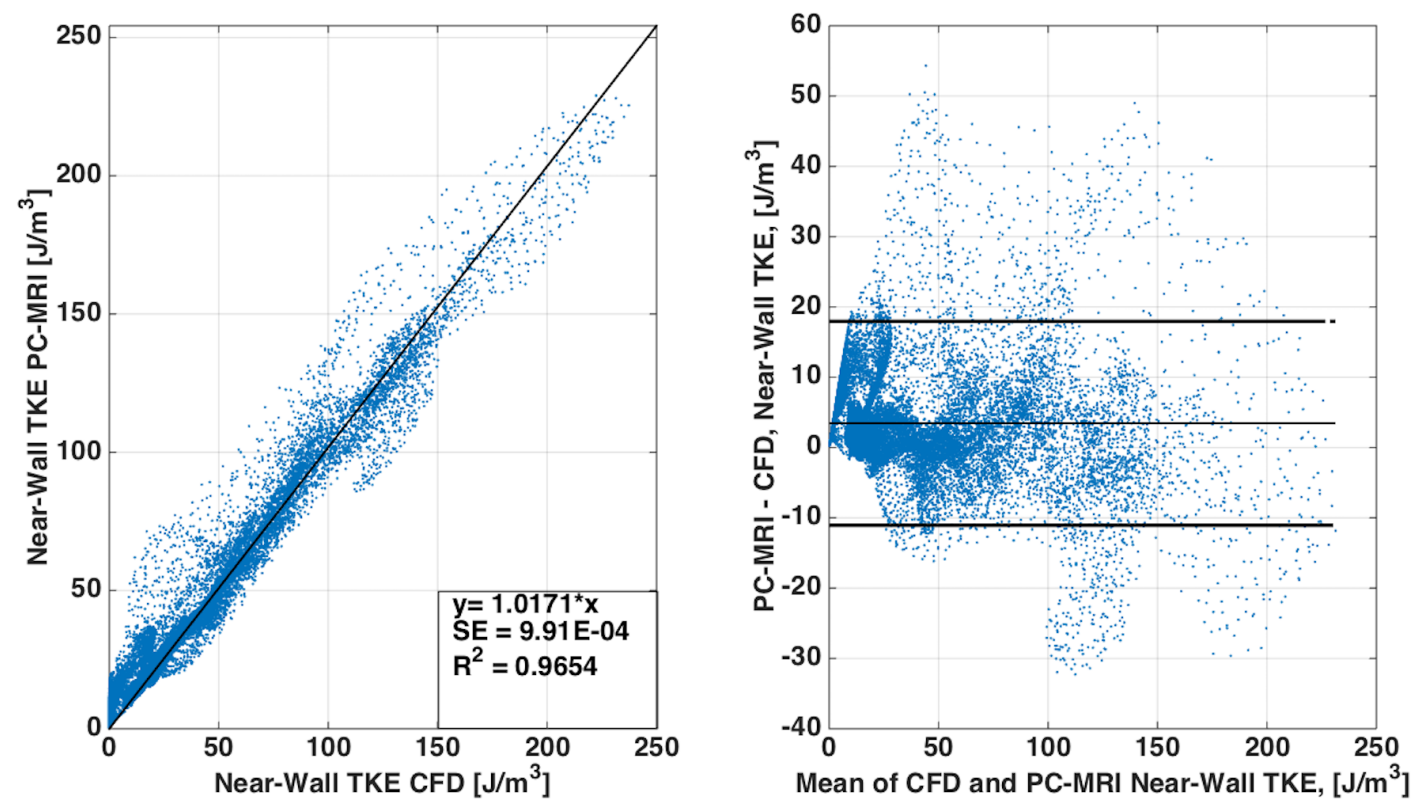

Figure 4 - Bland-Altman analysis of the MR-estimated near-wall TKE versus the CFD near-wall TKE in the CoA100 2.5mm model. There is good agreement with CFD, with a bias +/- 2SD of $15.4+/-31.7\left[\mathrm{~J} / \mathrm{m}^{3}\right]$. The linear regression had a slope of $1.25(\mathrm{p}<0.0001)$ and $\mathrm{R}^{2}$ of 0.90 . 

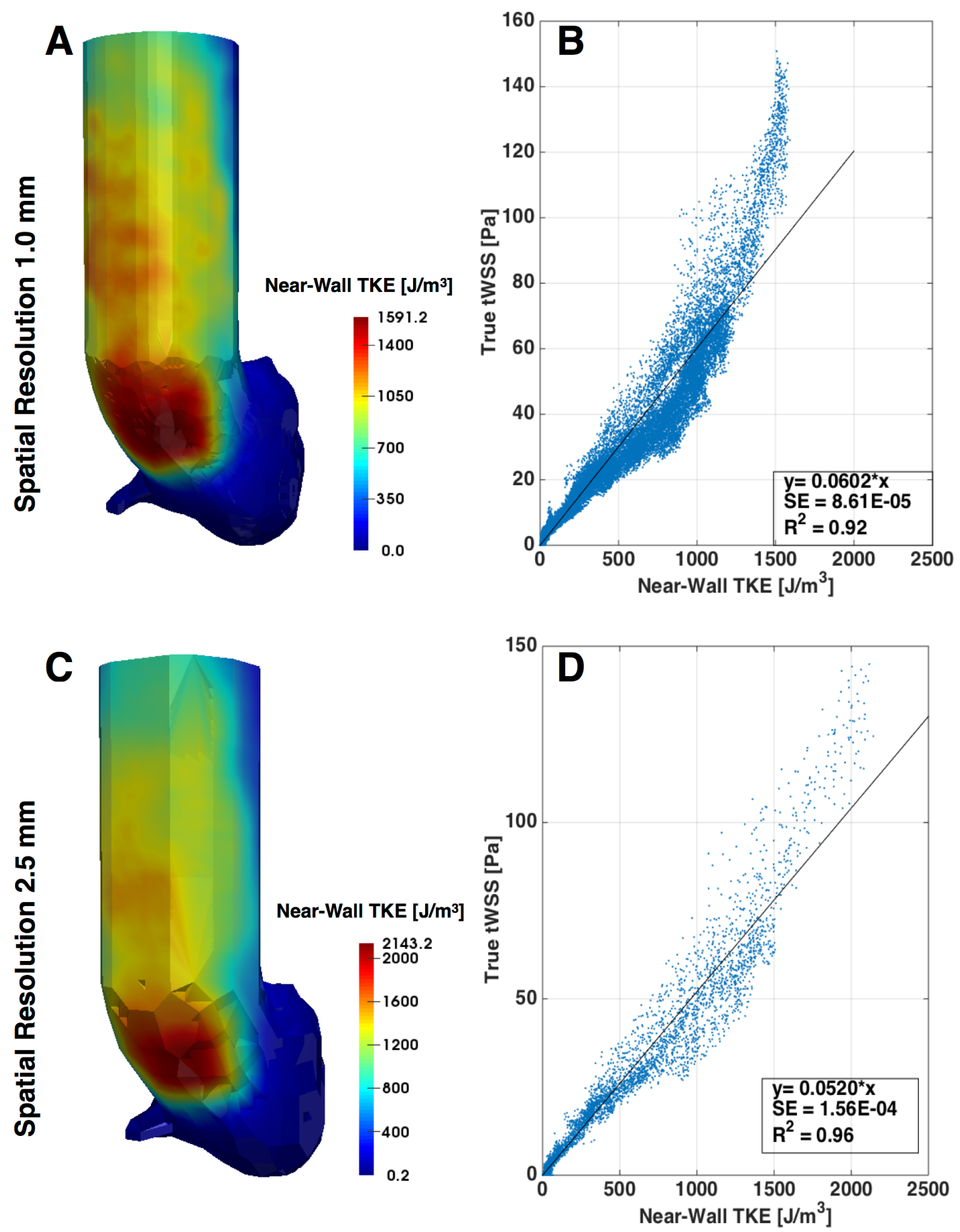

Figure 5 - Representative near-wall TKE estimation results for the AS100 model at spatial resolutions of 1 $\mathrm{mm}(\mathrm{A} \& \mathrm{~B})$ and $2.5 \mathrm{~mm}(\mathrm{C} \& \mathrm{D})$. A \& $\mathrm{C}$ show maps of near-wall TKE while B \& D show linear regression results for MR-estimated near-wall TKE versus CFD derived tWSS. The linear regression at 1 $\mathrm{mm}$ had $\mathrm{R}^{2}=0.90$, and $\mathrm{R}^{2}=0.93$ at $2.5 \mathrm{~mm}$. 

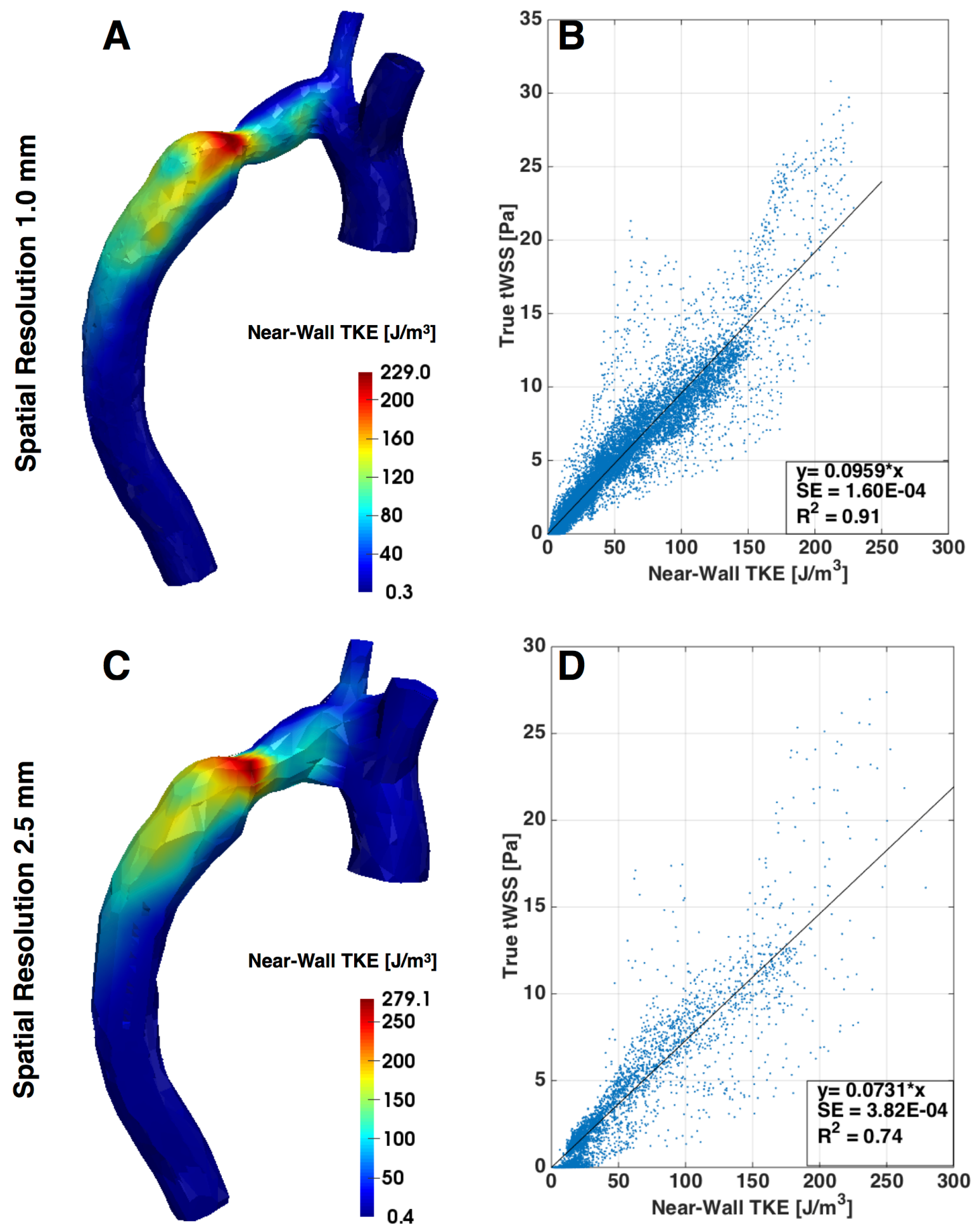

Figure 6 - Representative near-wall TKE estimation results for the CoA100 model at spatial resolutions of $1 \mathrm{~mm}(\mathrm{~A} \& \mathrm{~B})$ and $2.5 \mathrm{~mm}(\mathrm{C} \& \mathrm{D})$. A \& $\mathrm{C}$ show maps of near-wall TKE while B $\& \mathrm{D}$ show linear regression results for MR-estimated near-wall TKE versus CFD derived tWSS. The linear regression at $1 \mathrm{~mm}$ had $\mathrm{R}^{2}=0.90$, and $\mathrm{R}^{2}=0.81$ at $2.5 \mathrm{~mm}$. 